\title{
Comparison of diagnostic performance of different imaging modalities for TAVI-patients
}

\author{
Yang Chul Boering*, Mirja Neizel, Florian Bönner, Sebastian Grünig, Marc W Merx, Tobias Zeus, Stefan Steiner, \\ Jan Balzer, Malte Kelm, Burkhard Sievers \\ From 15th Annual SCMR Scientific Sessions \\ Orlando, FL, USA. 2-5 February 2012
}

\section{Background}

In contrast to surgical aortic valve replacement pre procedural assessment of aortic, aortic annulus diameter and prosthesis size is essential for successful percutaneous interventional valve therapy. Among different imaging modalities multislice-computed tomography (MSCT) and transesophageal echocardiography (TEE) are routinely used for patient screening. We sought to evaluate the diagnostic performance of cardiac magnetic resonance tomography (CMR) in this setting, and the difference in diameter measurements between all 3 imaging modalities.

\section{Methods}

30 patients (mean age $80 \pm 8$ years, 19 male) were underwent TEE (Philips iE33, Andover, USA), MSCT (64-slice Siemens Somatom, Forchheim, Germany), and CMR (Philips Achieva, Best, Netherlands) studies to assess enddiastolic diameters of aortic annulus, aortic bulbus and ascending aorta. In addition, minimal aortic valve area was assessed by TEE and CMR. CT data were retrospectively triggered and reconstruction was set to 60$75 \%$ of the cardiac phase. CMR-studies were prospectively triggered using a free breathing navigator for acquisition of a full 3D volume data set (cardiac phase 50-80\%). Subsequent image analysis for MSCT and CMR was performed using the vendor specific dedicated $3 \mathrm{D}$ analysis tools. TEE measurements were performed on $2 \mathrm{D}$ images of 3-chamber views.

\section{Results}

Aortic annulus diameter was $24.2 \pm 2.8 \mathrm{~mm}$ for MSCT, $23.36 \pm 2.4 \mathrm{~mm}$ for $\mathrm{CMR}$ and $22.6 \pm 2.01 \mathrm{~mm}$ for TEE $(\mathrm{p}<0.01)$. Aortic bulbus was measured at $33.71 \pm 3.6 \mathrm{~mm}$ on MSCT, $32.75 \pm 2.4 \mathrm{~mm}$ on CMR and $30.5 \pm 2.01 \mathrm{~mm}$ on TEE images $(\mathrm{p}<0.01)$. Ascending aortic diameter was $30.63 \pm 5.04 \mathrm{~mm}$ on MSCT, $28.43 \pm 4.11 \mathrm{~mm}$ on CMR, and $28.5 \pm 4.08 \mathrm{~mm}$ on TEE $(\mathrm{p}<0.01)$. Differences between measurements and imaging modalities were assessed by Bland Altman statistics. Mean SD for 1) aortic annulus measurements was $0.8 \pm 4.6 \mathrm{~mm}$ for TEE versus CMR and $1.1 \pm 3.7 \mathrm{~mm}$ for MSCT versus CMR, 2) aortic bulbus diameters $2.2 \pm 2.7 \mathrm{~mm}$ for TEE versus CMR and 1.0 \pm 3.7 $\mathrm{mm}$ for MSCT versus CMR, and 3) ascending aorta 0.9 $\pm 3.1 \mathrm{~mm}$ for TEE versus CMR and $2.2 \pm 3.5 \mathrm{~mm}$ for MSCT versus CMR. The minimal aortic valve area showed good agreement between CMR and TEE, standard deviation $0.04 \pm 0.41 \mathrm{sqcm}$.

\section{Conclusions}

Aortic diameter measurements based on MSCT images were significantly higher compared to CMR and TEE. The lowest diameters were consistently measured on TEE. Our findings may have a relevant clinical impact for decision making in pre procedural TAVI planning and work flow regarding size and type of aortic valve prosthesis.

\section{Funding}

No funding.

Published: 1 February 2012

doi:10.1186/1532-429X-14-S1-P97

Cite this article as: Boering et al: Comparison of diagnostic

performance of different imaging modalities for TAVI-patients. Journal of Cardiovascular Magnetic Resonance 2012 14(Suppl 1):P97.

Cardiology, Heinrich Heine University, Duesseldorf, Germany

๑ 2012 Boering et al; licensee BioMed Central Ltd. This is an open access article distributed under the terms of the Creative Commons 